\title{
REUTILIZACIÓN DE AGUA RESIDUAL PARA RIEGO DE JUDÍA: EFECTO DE LA CARGA FERTILIZANTE, MICROBIOLÓGICA Y DE ELEMENTOS TRAZA
}

\author{
$M^{a}$ Luz Segura, Emilio Martín, Sonia Martínez, Juana I. Contreras \\ IFAPA Almería, Consejería de Innovación Ciencia y Empresa \\ Junta de Andalucía \\ Autovía del Mediterráneo, salida 420, 04745 La Mojonera, Almería (España) \\ marial.segura.ext@juntadeandalucia.es
}

\begin{abstract}
Resumen: En este trabajo se estudió la respuesta del cultivo de judía verde al riego con agua residual de origen urbano. La experiencia se realizó sobre la variedad "Helda" desarrollada en condiciones de invernadero, suelo enarenado y sistema de riego por goteo. Los tratamientos consistieron en el riego con agua de origen subterráneo $\left(A_{S}\right)$ y residual ozonizada $\left(A_{\circ}\right)$. Se evaluaron los rendimientos de fruto, concentración de $\mathrm{N}$ total, mineral y $\mathrm{K}$ asimilable del suelo, concentración total de $\mathrm{N}$ y $\mathrm{K}$ en limbo foliar y parte aérea de la planta, contenido de coliformes totales y fecales del agua de riego, coliformes totales y Escherichia Coli en suelo, coliformes totales y Escherichia Coli del fruto, concentración de elementos traza del agua de riego y concentración de boro en extracto saturado de suelo y en limbo foliar de la planta. No hubo efectos significativos debido al tipo de agua sobre la respuesta productiva y nutricional del cultivo, con un ahorro de $\mathrm{N}$ y $\mathrm{K}$ en el tratamiento regado con agua residual ozonizada. El contenido de coliformes fecales del agua ozonizada no sobrepasó los niveles máximos exigidos para riego, no detectándose contaminación en suelo ni en fruto. La concentración de elementos traza no superó los niveles establecidos para su uso en regadío, a excepción del boro cuyos niveles deben ser controlados en el suelo y la planta.
\end{abstract}

\section{INTRODUCCIÓN}

El sistema agrícola en Almería se centra en la producción de cultivos hortícolas intensivos, con una superficie de invernaderos de 27.000 hectáreas (Arrojo, 2003). El cultivo de judía es uno de los más importantes con una superficie de 3.925 hectáreas y una producción en el año 2003 , de 61.900 toneladas, que reportaron aproximadamente 82 millones de euros (Consejería de Agricultura y Pesca, 2004). La escasez de precipitaciones, con una lluvia eficaz de unos 40-42 $\mathrm{mm}$ al año (Salinas, 2001), y la sobreexplotación y deterioro de los acuíferos, con un déficit hídrico estructural de $264 \mathrm{Hm}^{3}$ al año (Consejería de Agricultura y Pesca-Junta de Andalucía, 2002), son factores que limitan la expansión e incluso el sostenimiento de este sector productivo. La búsqueda y optimización de utilización de otras fuentes hídricas adicionales se enmarcan como uno de los objetivos prioritarios para el mantenimiento de los niveles productivos actuales. En este sentido la reutilización de aguas residuales para riego de los cultivos se presenta como una alternativa, puesto que proporciona la llave para la eficiente y efectiva utilización de los recursos limitados de agua y aporta un nuevo valor debido a la carga fertilizante que contiene (Shahalam et al., 1998). Además, la reutilización de aguas residuales se presenta como una opción atractiva para la protección del medio ambiente proporcionando un recurso de agua adicional (Xu et al., 2002) que previene la contaminación por vertido al medio natural y elimina riesgos para la salud (Brenner et al., 2000).

Numerosos trabajos demuestran la eficiencia de estos efluentes para riego de cultivos tales como plantas forrajeras (Bole y Bell, 1978), cereales (Al-Jaloud et al., 1993, Montserrat, 1993), algodón (Bielorai et al., 1984), cítricos (Kale y Bal, 1987, Koo y Zekri, 1989, Maurer et al., 1995) y cultivos hortícolas (Basiouny, 1984, Rosas et al., 1984, Kirkham, 1986, Neilsen et al., 1989, Shahalam et al., 1998, Maloupa et al., 1999, Segura et al., 2001). No obstante, la reutilización de las aguas residuales puede pre- 
sentar ciertas limitaciones de uso debido a la presencia de microorganismos patógenos (Escherichia coli, Salmonella sp., Shigella sp., etc.) y de elementos traza. Los primeros, bajo determinadas condiciones, pueden sobrevivir durante periodos de tiempo variables, con cierto potencial de infección tanto para la explotación agrícola como para el consumidor final (Pescod, 1992) con lo cual será imprescindible proceder a la descarga microbiológica, sometiéndolas a un grado de depuración que será función de la carga contaminante, de la sensibilidad del medio receptor en caso de vertido o del destino que vayan a tener en caso de aprovechamiento posterior (Pérez y Vallverdú, 1997). Respecto a los elementos traza, un aporte incontrolado de éstos al suelo puede producir una acumulación excesiva siendo difícil eliminarlos (Page et al., 1981, Hussain y Al-Saati, 1999). Niveles elevados de elementos traza en el agua pueden producir por un lado efectos tóxicos sobre las plantas sensibles a los mismos, y por otro, un elevado riesgo para las personas o animales que los consuman a concentraciones excesivas en los tejidos vegetales (Ramos, 1997). Entre los elementos traza que puede contener el agua residual caben destacar el B, Cd, Cu, Ni y Zn, debido a sus efectos fitotóxicos potenciales (Page et al., 1981).

La desinfección del agua residual con ozono, producido "in situ" a partir del oxígeno contenido en el aire es una técnica muy eficiente y económicamente viable si el uso del agua es para riego de cultivos hortícolas (Pérez y Vallverdú, 1997). El ozono es un desinfectante fuerte con un alto potencial de oxidación que resulta ser uno de los más efectivos y más usados para la inactivación de patógenos en el tratamiento de agua potable (Langlais et al., 1991).

El riego con agua residual ozonizada de cultivos hortícolas moderadamente tolerantes a la salinidad, como melón y tomate, no limita el desarrollo de la planta ni la producción de frutos. Debido a la carga fertilizante que posee, permite ahorros de fertilizantes nitrogenados y potásicos entre un 10 y un $40 \%$, según elemento y cultivo (Segura et al., 2000). La información sobre la respuesta de cultivos más sensibles a la salinidad, como es la judía verde, al riego con el efluente es escasa. Respecto a la salinidad del agua de riego este cultivo es uno de los que presenta mayor sensibilidad (Ayers y Westcot, 1985) si bien Martínez (1987) determinó una mayor tolerancia cuando se desarrolla en condiciones de suelo enarenado. Desde el punto de vista nutricional, según López-Gálvez et al. (1991), el cultivo de judía desarrollado en condiciones similares tiene unas necesidades en $\mathrm{N}$ y $\mathrm{K}$ de 4,5 y $7,0 \mathrm{~g} \mathrm{~m}^{-2}$ $\mathrm{Kg}^{-1}$ fruto. El riego del cultivo con el efluente cubre las necesidades de $\mathrm{N}$ en determinadas épocas (desarrollo vegetativo y recolección) lo cual puede incidir en una reducción de aporte de fertilizantes minerales y del impacto ambiental asociado al efecto residual de los mismos (Segura et al., 2003).

El objetivo de este trabajo ha sido el estudio de la respuesta del cultivo de judía verde de invernadero al riego, utilizando agua residual de origen urbano y considerando la carga fertilizante, el contenido microbiológico y los elementos traza del efluente.

\section{MATERIALES Y MÉTODOS}

La experiencia se realizó sobre una superficie de $500 \mathrm{~m}^{2}$ en un invernadero de arco simétrico con cubierta de polietileno, situado en el Centro de Investigación y Formación Agraria (I.F.A.P.A. Junta de Andalucía), situado en el término municipal de La Cañada de San Urbano (Almería). El sistema de cultivo fue suelo enarenado mayoritariamente utilizado en la zona productora, de textura franco-arenosa. La Tabla 1 muestra las propiedades químicas del suelo. La mayor concentración de $\mathrm{N}$ (forma nítrica y amoniacal) y $P$ asimilable del suelo en el tratamiento $A_{O}$, al inicio de la experiencia, se debió a efectos residuales de experiencias anteriores en las que se testaron otros cultivos hortícolas.

\begin{tabular}{|c|c|c|c|c|c|c|c|c|}
\hline \multirow[t]{2}{*}{ Tratamiento } & \multirow{2}{*}{$\begin{array}{c}\text { M.O. } \\
(\%)\end{array}$} & \multirow{2}{*}{$\begin{array}{c}\text { Ntotal } \\
(\%)\end{array}$} & $\mathbf{N}\left(\mathrm{NO}_{3}^{-}\right)$ & $\mathbf{N}\left(\mathrm{NH}_{4}^{+}\right)$ & $\mathbf{P}_{\text {asim. }}$ & $\mathrm{K}_{\mathrm{asim}}$ & Ca $a_{\text {asim. }}$ & $\mathbf{M g}_{\text {asim }}$ \\
\hline & & & \multicolumn{6}{|c|}{$\left(\mathrm{mg} \mathrm{kg}^{-1}\right)$} \\
\hline $\begin{array}{l}A_{S} \\
A_{O} \\
\text { ANOVA }\end{array}$ & $\begin{array}{c}0,66 \\
0,67 \\
\text { NS }\end{array}$ & $\begin{array}{c}0,03 \\
0,02 \\
\text { NS }\end{array}$ & $\begin{array}{c}6,66 \\
10,35 \\
\mathrm{~S}\end{array}$ & $\begin{array}{c}7,18 \\
9,80 \\
\mathrm{~S}\end{array}$ & $\begin{array}{c}108 \\
176 \\
S\end{array}$ & $\begin{array}{l}227 \\
215 \\
\text { NS }\end{array}$ & $\begin{array}{c}1110 \\
1368 \\
\text { NS }\end{array}$ & $\begin{array}{l}204 \\
209 \\
\text { NS }\end{array}$ \\
\hline
\end{tabular}

Tabla 1. Análisis de caracterización del suelo. NS indica que no existe diferencia significativa entre tratamientos; $\mathrm{S}$ indica diferencias significativas entre tratamientos. M.O.: Materia orgánica oxidable. $A_{S}$ : agua subterránea y $A_{O}$ : agua residual ozonizada 
El cultivo de judía cv. Helda, se sembró en siembra directa sobre el suelo enarenado el $10 / 09 / 2001$, a una densidad de plantación de 3,17 plantas $\mathrm{m}^{-2}$. El número de plantas por tratamiento fue de 396. Se estableció un diseño experimental en bloques completos al azar compuesto por dos tratamientos y cuatro repeticiones por tratamiento. Los tratamientos consistieron en dos tipos de agua de riego, origen subterráneo $\left(A_{S}\right)$ y residual ozonizada $\left(A_{O}\right)$.

El agua de origen subterráneo fue la habitualmente utilizada en la zona productora y el agua residual ozonizada procedía de la Estación Depuradora de Aguas Residuales de Almería y fue sometida a un tratamiento terciario en la Planta de Ozono localizada en Viator (Almería). Las propiedades químicas de las dos fuentes de riego se especifican en la Tabla 2.

\begin{tabular}{lcc}
\hline & $\mathbf{A}_{\mathbf{S}}$ & $\mathbf{A}_{\mathbf{O}}$ \\
\hline $\mathrm{pH}$ & 7,58 & 7,63 \\
$\mathrm{C} . \mathrm{E}\left(\mathrm{dS} \mathrm{m}^{-1}\right)$ & 1,63 & 1,82 \\
$\mathrm{CO}_{3}^{2-}$ & 0,13 & 0,61 \\
$\mathrm{HCO}_{3-}$ & 1,94 & 7,45 \\
$\mathrm{Cl}^{-}$ & 7,58 & 6,85 \\
$\mathrm{SO}^{2-}$ & 7,34 & 3,83 \\
$\mathrm{~N}^{-} \mathrm{NO}^{3-}$ & 0,94 & 0,27 \\
$\mathrm{~N}^{3-} \mathrm{NH}_{4}^{+}\left(\mathrm{mEq} \mathrm{L}^{-1}\right)$ & 0,00 & 3,27 \\
$\mathrm{Ca}^{2+}$ & 4,50 & 3,41 \\
$\mathrm{Mg}^{2+}$ & 5,16 & 3,92 \\
$\mathrm{Na}^{+}$ & 7,88 & 8,33 \\
$\mathrm{~K}^{+}$ & 0,14 & 0,52 \\
$\mathrm{P}$ & 0,00 & 0,37 \\
$\mathrm{RAS}$ & 3,58 & 4,36 \\
$\mathrm{Clasificación}$ Riverside & $\mathrm{C}_{3} \mathrm{~S}_{1}$ & $\mathrm{C}_{3} \mathrm{~S}_{1}$ \\
\hline
\end{tabular}

Tabla 2. Análisis químico del agua de riego

Las dos aguas presentan $\mathrm{pH}$ similar y según Normas Riverside su clasificación es $\mathrm{C}_{3} \mathrm{~S}_{1}$, pero difieren en su composición química. Así el agua residual ozonizada contiene mayor concentración de bicarbonatos, nitrógeno amoniacal, fósforo y potasio que el agua convencional, y menor concentración de nitratos, sulfatos, calcio y magnesio (Tabla 2).

Se aplicó una fertilización nitrógeno-potásica, mediante fertirrigación, similar en los dos tratamientos y basada en las extracciones del cultivo determinadas para las condiciones locales según López-Gálvez et al. (1991). Para obtener disoluciones de riego con niveles similares de $\mathrm{N}$ y $\mathrm{K}$ en ambos tratamientos se consideró la concentración de éstos en el agua de riego. La cantidad de $\mathrm{N}$ y $\mathrm{K}$ aportada al cultivo a lo largo del ciclo se indica en la Figura 1. No se aplicó $P$ debido a su elevada concentración en el suelo (Tabla 1) y las cantidades de $\mathrm{Ca}, \mathrm{Mg}$ y $\mathrm{S}$ aportadas fueron las de la propia fuente de riego.

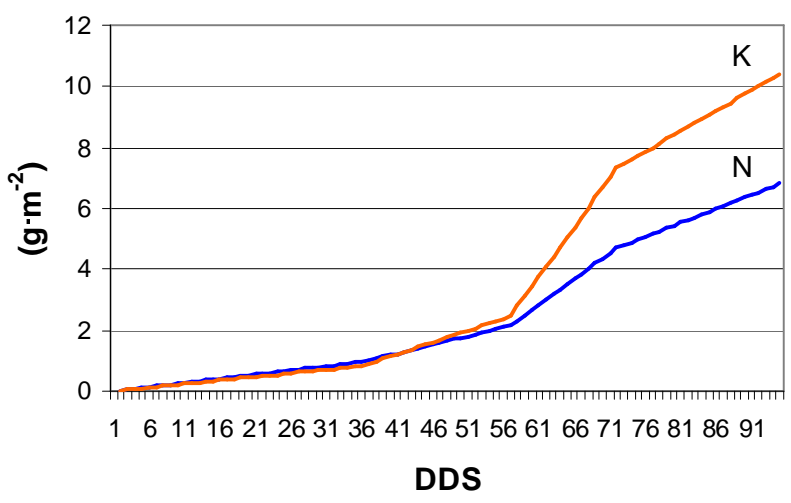

Figura 1. Nitrógeno $(\mathrm{N})$ y potasio $(\mathrm{K})$ aportados al cultivo de judía verde. DDS: Días Desde la Siembra

Las parcelas experimentales estaban separadas entre sí por medio de una lámina de polietileno enterrada hasta una profundidad de $30 \mathrm{~cm}$, para evitar el paso de solución del suelo de una parcela a otra. El sistema de fertirriego estaba compuesto por un programador automatizado, cuatro tanques de abonado y dos redes de distribución por goteo independientes, una para el agua de origen subterráneo $\left(A_{S}\right)$ y otra para el agua residual ozonizada $\left(A_{O}\right)$. El volumen de riego se estableció en base a las estimaciones de la evapotranspiración del cultivo $\left(E T_{c}\right.$ ) (Fernández et al., 2000) y medidas tensiométricas del suelo en la zona radical de las plantas $(15 \mathrm{~cm}$ de profundidad). El volumen total de agua aportado al cultivo en cada uno de los tratamientos fue de $130 \mathrm{~L} \mathrm{~m}^{-2}$.

Se evaluaron los rendimientos de fruto, recolectados durante 42 días, siguiendo la normativa europea de calidad y comercialización (Reglamento CEE 912/2001). En suelo se determinó la concentración de $\mathrm{N}$ total, mineral (nitrato y amonio) y $\mathrm{K}$ en forma asimilable, a los 86 días desde la siembra. En la planta se determinó la concentración total de $\mathrm{N}$ y $\mathrm{K}$ en limbo foliar durante el ciclo de cultivo, para lo cual se tomaron hojas maduras de diez plantas de cada una de las parcelas experimentales. La concentración total de $\mathrm{N}$ y $\mathrm{K}$ de la parte aérea de la planta se determinó al finalizar la experiencia, para lo cual se tomaron tres plantas por parcela experimental, las cuales se sometieron a una desecación en estufa de aire forzado a $70{ }^{\circ} \mathrm{C}$, hasta peso constante, determinándose posteriormente materia seca y concentración de $\mathrm{N}$ y $\mathrm{K}$. Las determinaciones analíticas en suelo, agua y planta se realizaron según los Métodos Oficiales (M.A.P.A., 1994). 
Mensualmente se determinó el contenido de coliformes totales y fecales del agua de riego. A los 92 días desde la siembra (DDS) se determinó el contenido de coliformes totales y Escherichia Coli en los primeros $5 \mathrm{~cm}$ de la capa de arena del tratamiento regado con agua residual ozonizada, así como el contenido de coliformes totales y Escherichia Coli del fruto en contacto con el suelo de este mismo tratamiento. Las técnicas utilizadas fueron las de filtración en membrana y Número Más Probable (A.P.H.A. et al., 1992). La concentración de elementos traza (B, Fe, Cu, Zn, Al, Mn, Ni, Mo, Cr, Pb, y Cd) del agua de riego, se determinó mediante espectrometría de emisión de plasma (Soltanpour, 1991). Durante el ciclo de cultivo (desarrollo vegetativo, floración-fructificación y plena recolección) se determinó la concentración de boro en extracto saturado de suelo y en limbo foliar de la planta, según los Métodos Oficiales de Análisis (M.A.P.A., 1994).

El análisis estadístico de datos se realizó mediante el análisis de la varianza (ANOVA) y la comparación de medias con el test de Mínima Diferencia Significativa con un nivel de significación del $5 \%$.

\begin{tabular}{ccc}
\hline Tratamiento & $\begin{array}{c}\text { Producción } \\
\left(\mathrm{kg} \mathrm{m}^{-2}\right)\end{array}$ & $\begin{array}{c}\mathbf{N}^{\circ} \text { vainas } \\
\mathrm{m}^{-2}\end{array}$ \\
\hline $\mathrm{A}_{\mathrm{S}}$ & 1,059 & 77,68 \\
$\mathrm{~A}_{\mathrm{O}}$ & 1,159 & 89,70 \\
ANOVA & NS & NS \\
\hline
\end{tabular}

Tabla 3. Rendimientos totales y número de vainas de judía. NS indica que no existen diferencias significativas entre tratamientos

\section{RESULTADOS Y DISCUSIÓN}

\section{Rendimiento del cultivo}

No se obtuvieron diferencias significativas entre tratamientos en la producción y número de vainas de judía (Tabla 3), siendo la producción total obtenida, en ambos tratamientos, del orden de $1100 \mathrm{~g} \mathrm{~m}^{-2}$. Los valores de producción obtenidos son similares a los registrados en la provincia de Almería (Consejería de Agricultura y Pesca, 2004).

\section{Concentración de $\mathrm{N}$ total e inorgánico y $\mathrm{K}$ asimilable del suelo}

La concentración de $\mathrm{N}$ total, $\mathrm{N}$ mineral (nitrato y amonio) y $\mathrm{K}$ asimilable del suelo, obtenidos al finalizar el ciclo de cultivo, se presenta en la Tabla 4. No hubo diferencias significativas entre tratamientos en la concentración de nitrógeno total, nítrico y amoniacal. Tampoco se obtuvieron diferencias significativas entre tratamientos en la concentración de potasio asimilable del suelo. Los resultados obtenidos muestran, respecto a los valores iniciales (Tabla 1 ), un ligero aumento de las concentraciones de $\mathrm{N}^{-\mathrm{NO}_{3}^{-}}$y K , siendo dichos niveles medios y normales, respectivamente (Soltanpour, 1991; Cadahía, 1997). El efecto similar producido por los dos tipos de agua, sobre los niveles de $\mathrm{N}$ y $\mathrm{K}$ asimilables del suelo justifica el que se haya obtenido la misma respuesta productiva del cultivo en ambos tratamientos.

\section{Concentración de $\mathrm{N}$ y $\mathrm{K}$ en la planta}

Las Tablas 5 y 6 muestran la concentración de $\mathrm{N}$ y $\mathrm{K}$ en limbo foliar y en la parte aérea de la planta, respectivamente. La concentración de $\mathrm{N}$ y $\mathrm{K}$ en limbo foliar fue similar en ambos tratamientos. La concentración en $\mathrm{N}$ aumentó en la última fase del ciclo, si bien los niveles de este elemento durante la experiencia se mantuvieron dentro de los considerados normales para este tipo de cultivo (Casas y Casas, 1999). La concentración de $\mathrm{K}$, disminuyó a los 53 días desde la siembra (floración-fructificación), manteniéndose en este nivel hasta el final del ciclo. Los valores obtenidos se sitúan dentro del rango de normalidad (Casas y Casas, 1999) y son similares a los registrados por Hita (2002) en condiciones similares de desarrollo.

En cuanto a la concentración total de $\mathrm{N}$ y $\mathrm{K}$ en la parte aérea de la planta, expresada como producto de la concentración del elemento por el

\begin{tabular}{lcccc}
\hline Tratamiento & $\mathbf{N}$ total & $\mathbf{N}\left(\mathbf{N O}_{3}^{-}\right)$ & $\mathbf{N}\left(\mathbf{N H}_{4}^{+}\right)$ & $\mathbf{K}_{\text {asim. }}$ \\
\cline { 3 - 5 } & $(\%)$ & \multicolumn{3}{c}{$\left(\mathrm{mg} \mathrm{kg}^{-\mathbf{1}}\right)$} \\
\hline $\mathrm{A}_{\mathrm{S}}$ & 0,07 & 10,32 & 1,96 & 255 \\
$\mathrm{~A}_{\mathrm{O}}$ & 0,07 & 11,34 & 2,80 & 247 \\
ANOVA & $\mathrm{NS}$ & $\mathrm{NS}$ & $\mathrm{NS}$ & $\mathrm{NS}$ \\
\hline
\end{tabular}

Tabla 4. Efecto de los tratamientos sobre la concentración de $\mathrm{N}$ y $\mathrm{K}$ del suelo. NS indica que no existen diferencias significativas entre tratamientos 


\begin{tabular}{cccc}
\hline DDS & Tratamiento & $\mathbf{N}$ & $\mathbf{K}$ \\
\hline \multirow{2}{*}{35} & $\mathrm{~A}_{\mathrm{S}}$ & 4,43 & 3,11 \\
& $\mathrm{~A}_{\mathrm{O}}$ & 4,63 & 2,97 \\
& ANOVA & NS & NS \\
\hline \multirow{2}{*}{53} & $\mathrm{~A}_{\mathrm{S}}$ & 4,46 & 2,23 \\
& $\mathrm{~A}_{\mathrm{O}}$ & 4,74 & 2,57 \\
& ANOVA & $\mathrm{NS}$ & $\mathrm{NS}$ \\
\hline \multirow{2}{*}{80} & $\mathrm{~A}_{\mathrm{S}}$ & 5,19 & 2,48 \\
& $\mathrm{~A}_{\mathrm{O}}$ & 5,41 & 2,557 \\
& ANOVA & NS & NS \\
\hline
\end{tabular}

Tabla 5. Concentración de $\mathrm{N}$ y K ( \% s.m.s.: porcentaje sobre materia seca) en limbo foliar. DDS: días desde la siembra. NS indica que no existen diferencias significativas entre tratamientos

peso seco (Tabla 6), no existieron diferencias significativas entre ambos tratamientos, extrayendo la parte aérea del cultivo $8,2 \mathrm{~g}$ de nitrógeno por $\mathrm{m}^{2}$ y $10,6 \mathrm{~g}$ de potasio por $\mathrm{m}^{2}$ como media de las dos variables consideradas en el estudio. La concentración de nitrógeno y de potasio extraído por la planta fue mayor que la obtenida en cultivos de judía en condiciones de desarrollo similares, por López-Gálvez et al. (1991) e Hita (2002).

\section{Ahorro de fertilizantes}

La concentración de nitrógeno y potasio del agua residual ozonizada supuso un ahorro importante de fertilizantes minerales nitrogenados y potásicos, al aportar el efluente un $95 \%$ del $\mathrm{N}$ y $25 \%$ del $\mathrm{K}$ requerido por el cultivo (Figura 2 ). El agua de origen subterráneo aplicó un 25 y $7 \%$ de $\mathrm{N}$ y $\mathrm{K}$, respectivamente.

\section{Contenido microbiológico del agua y efecto sobre el suelo y el fruto}

La Tabla 7 muestra la concentración de coliformes totales y fecales del agua. El agua residual ozonizada contiene una carga de coliformes totales elevada, apreciándose una gran variabilidad a lo largo de la experiencia, lo cual puede deberse al manejo del sistema de distribución (Crook, 1991; Contreras, 2001). Sin embargo el contenido de coliformes fecales no superó el nivel máximo de 1000 Unidades Formadoras de Colonias (U.F.C.) $100 \mathrm{~mL}^{-1}$, recomendado por la Organización Mundial de la Salud (O.M.S., 1989) para vegetales de consumo en crudo.

La Tabla 8 muestra los resultados del análisis microbiológico realizado en suelo y fruto, a los 92 días después de la siembra del cultivo (DDS). En el tratamiento regado con agua residual ozonizada se obtuvo unos niveles de coliformes totales y de Escherichia coli inferiores al límite de

\begin{tabular}{ccccc}
\hline Tratamiento & $\begin{array}{c}\mathbf{N} \text { total en planta } \\
\left(\mathrm{g} \mathrm{m}^{-2}\right)\end{array}$ & $\begin{array}{c}\text { Eficiencia de } \mathbf{N} \\
(\%)\end{array}$ & $\begin{array}{c}\mathbf{K} \text { total en planta } \\
\left(\mathrm{g} \mathrm{m}^{-2}\right)\end{array}$ & $\begin{array}{c}\text { Eficiencia de } \mathbf{K} \\
(\%)\end{array}$ \\
\hline $\mathrm{A}_{\mathrm{S}}$ & 7,96 & 117 & 10,13 & 97 \\
$\mathrm{~A}_{\mathrm{O}}$ & 8,33 & 122 & 10,97 & 105 \\
ANOVA & NS & NS & NS & \\
\hline
\end{tabular}

Tabla 6. Concentración total de $\mathrm{N}$ y $\mathrm{K}$ en planta y eficiencia absorbente de elemento. NS.: Indica que no existen diferencias significativas entre tratamientos

\begin{tabular}{|c|c|c|}
\hline & NITRÓGENO & POTASIO \\
\hline $\mathbf{A}_{\mathbf{S}}$ & $\overbrace{\substack{\text { Fertilizantes } \\
74,9 \%}}^{\text {Agua }} 25,1 \%$ & Fertilizantes \\
\hline $\mathbf{A}_{\mathbf{O}}$ & $\underset{\text { Agua }}{\mathbf{9 4 , 7 \%}}$ & Fertilizantes \\
\hline
\end{tabular}

Figura 2. Porcentaje de $\mathrm{N}$ y $\mathrm{K}$ respecto al total, aportados por el agua y los fertilizantes 


\begin{tabular}{cccccccc}
\hline & \multicolumn{3}{c}{$\mathbf{A}_{\mathbf{S}}$} & \multicolumn{3}{c}{$\mathbf{A}_{\mathbf{O}}$} \\
\cline { 2 - 7 } & Media & Máximo & Mínimo & Media & Máximo & Mínimo \\
\hline Coliformes Totales & 0 & 0 & 0 & 4700 & 13000 & 230 \\
Coliformes Fecales & 0 & 0 & 0 & 50 & 70 & 40 \\
\hline
\end{tabular}

Tabla 7. Análisis microbiológico (Unidades Formadoras de Colonias (U.F.C.) 100 $\mathrm{mL}^{-1}$ ) del agua de riego. $\mathrm{N}=3$

detección de la técnica utilizada (3 microorganismos por gramo) tanto en suelo como en fruto, por ello, no se detectó presencia de microorganismos indicadores de contaminación fecal.

\begin{tabular}{ccc}
\hline & $\begin{array}{c}\text { Coliformes Totales } \\
\left(\mathbf{n}^{\circ} \mathbf{g}^{-\mathbf{1}}\right)\end{array}$ & $\begin{array}{c}\boldsymbol{E} \text {. } \text { Coli } \\
\left(\mathbf{n}^{\circ} \mathbf{g}^{-\mathbf{1}}\right)\end{array}$ \\
\hline Suelo & $<3$ & $<3$ \\
Fruto & $<3$ & $<3$ \\
\hline
\end{tabular}

Tabla 8. Análisis microbiológico del suelo y fruto del tratamiento $A_{O}$ a los 92 DDS

\section{Concentración de elementos traza del agua de riego}

La Tabla 9 muestra la concentración media de elementos traza de las aguas de riego. El agua residual ozonizada $\left(A_{O}\right)$ presentó mayor concentración de $\mathrm{B}, \mathrm{Al}, \mathrm{Mn}$ y $\mathrm{Cr}$ y menor concentración de $\mathrm{Cu}$ y $\mathrm{Zn}$, que el agua de origen subterráneo $\left(A_{S}\right)$. Las concentraciones de los elementos traza analizados, no superaron los niveles máximos establecidos por Ayers y Westcot (1985) para aguas de uso agrícola, excepto el boro, cuya concentración en el agua residual ozonizada, superó el nivel máximo recomendado de $0,75 \mathrm{ppm}$, si bien el agua de origen subterráneo mantuvo sus niveles en el límite. Por otra parte, estos valores de boro en el agua son normales en esta zona del Bajo Andarax ya que, según Martínez et al. (1990) se han registrado valores de boro en el agua de la zona de hasta 6,9 ppm. Esto puede deberse al paso del agua por rocas derivadas de sedimentos marinos o de una roca madre rica en boro (Alarcón, 2001). En el caso del agua residual ozonizada se debe a que su origen deriva del aporte de detergentes y jabones de uso doméstico (Rojo, et al., 1996).

Efecto de la concentración de boro del agua de riego sobre los niveles de este elemento en suelo y planta

La Figura 3 muestra la concentración de boro soluble medido en extracto saturado del suelo. La mayor concentración de boro del agua residual ozonizada, produjo un aumento significativo de la concentración del elemento en el suelo,

\begin{tabular}{cccccccccccc}
\hline Tratamiento & $\mathbf{B}$ & $\mathbf{F e}$ & $\mathbf{C u}$ & $\mathbf{Z n}$ & $\mathbf{A l}$ & $\mathbf{M n}$ & $\mathbf{N i}$ & $\mathbf{M o}$ & $\mathbf{C r}$ & $\mathbf{P b}$ & $\mathbf{C d}$ \\
\hline $\mathrm{A}_{\mathrm{S}}$ & 783 & 354 & 57 & 42 & 24 & 5,9 & 3,6 & 2,4 & 1,3 & 0,78 & 0,12 \\
$\mathrm{~A}_{\mathrm{O}}$ & 1043 & 349 & 38 & 19 & 25 & 33 & 4,0 & 1,6 & 2,1 & 1,22 & 0,11 \\
ANOVA & $\mathrm{S}$ & NS & $\mathrm{S}$ & $\mathrm{S}$ & $\mathrm{S}$ & $\mathrm{S}$ & NS & NS & $\mathrm{S}$ & NS & NS \\
\hline
\end{tabular}

Tabla 9. Concentración media de elementos traza $\left(\mu g L^{-1}\right)$ del agua de riego. Cada valor es medía de tres determinaciones. $S$ indica que existen diferencias significativas entre tratamientos; NS indica que no existen diferencias significativas entre tratamientos

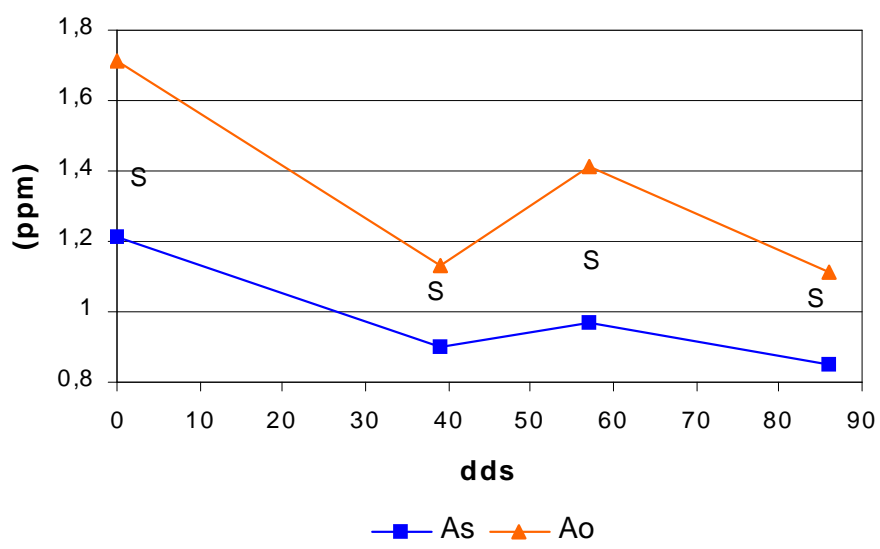

Figura 3. Concentración de $\mathrm{B}$ del extracto saturado de suelo. $\mathrm{S}$ indica que existen diferencias significativas entre tratamientos 


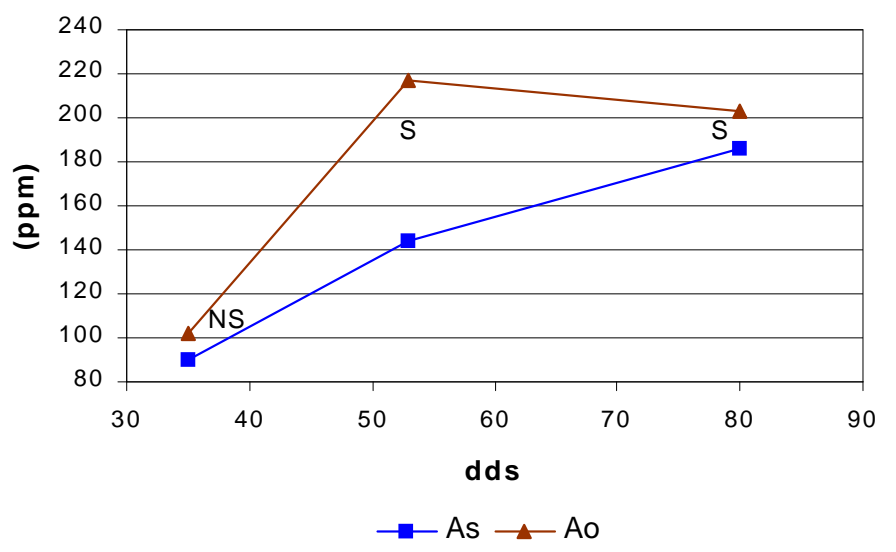

Figura 4. Concentración de B en limbo foliar. NS indica que no existen diferencias significativas entre tratamientos. $S$ indica que existen diferencias significativas entre tratamiento

oscilando sus valores entre 1,1 y $1,7 \mathrm{ppm}$. Para el tratamiento $A_{S}$ la concentración de $B$ osciló entre 0,9 y $1,2 \mathrm{ppm}$. Ambos tratamiento superaron el nivel máximo recomendado (1 ppm) para este cultivo (Ayers y Westcot, 1985).

La Figura 4 muestra la concentración de boro total en limbo foliar. Se obtuvieron diferencias significativas entre tratamientos en la concentración de boro, siendo mayor la concentración de boro en el tratamiento regado con agua residual ozonizada, de acuerdo a los niveles de B soluble del suelo. Los valores obtenidos en la planta en $A_{O}$, a los 53 y 80 DDS, superaron los 193 ppm de boro establecidos por Casas y Casas (1999) como niveles críticos para el cultivo de judía en las condiciones locales de cultivo, si bien no llegó a manifestarse de forma visual síntomas asociados a problemas de toxicidad en la planta debido quizás a que esta situación no fue constante durante todo el ciclo de cultivo.

\section{CONCLUSIONES}

El riego con agua residual ozonizada produjo efectos similares al agua de origen subterráneo sobre la respuesta productiva y nutricional del cultivo de judía. Con un ahorro de $\mathrm{N}$ y $\mathrm{K}$ respecto al agua de origen subterráneo, del $70 \%$ y $19 \%$ respectivamente.

El contenido de coliformes fecales del agua residual ozonizada, durante el tiempo de duración del ensayo, no sobrepasó los niveles máximos exigidos para riego, no detectándose contaminación en suelo ni en fruto.

La concentración de elementos traza del agua residual ozonizada no supera los niveles establecidos para su uso en regadío, a excepción del boro cuyos niveles deben ser controlados en suelo y planta.

\section{AGRADECIMIENTOS}

Este trabajo ha sido financiado por la D.G.I.A. Consejería de Agricultura y Pesca. Junta de Andalucía (proyecto P.I.A.-12-01-3).

\section{REFERENCIAS}

Alarcón, A. (2001). El boro como nutriente esencial. Horticultura septiembre, 155, 36-43.

Al-Jaloud, A., Hussain, G., Al-Saati, A.J. y Karimullah, S., (1993). Effect of wastewaters on plant growth and soil properties. Arid Soil Research Rehabilitation 7, 173-179.

APHA, AWWA y WPCF, (1992). Métodos Normalizados para el análisis de aguas potables y residuales. Ed. Díaz de Santos, S.A., Madrid (España).

Arrojo, P., (2003). El plan hidrológico nacional. Una cita frustrada con la historia. Integral (ed.), Barcelona (España).

Ayers, R.S. y Westcot, D.W., (1985). Irrigation water quality criteria. Irrigatión with Reclaimed Municipal Wastewater- A Guidance Manual. Report No 84-1, Ed. G.S. Pettygrove y T. Asano, Calif. State Water Resources Control Board, Sacramento, California (USA).

Basiouny, F.M., (1984). The use of municipal treated effluent for peach tree irrigation. Proceeding of Florida State Horticultural Society, 97, 345-347.

Bielorai, H., Vaisman, I. y Felgini, A., (1984). Drip irrigation of cotton with treated municipal effluents: I. Yield response. Journal of Environmental Quality, 13, 231-234. 
Bole, J.B. y Bell, R.G., (1978). Land application of municipal wastewater: yield and chemical composition of forage crops. Journal of Environmental Quality, 7, 222226

Brenner, A., Shandalov, S., Messalem, R., Yakirevich, A., Oron, G. y Rebhun, M., (2000). Wastewater reclamation for agricultural reuse in Israel: trends and experimental results. Water, Air and Soil Pollution, 123, 167-182.

Cadahía, C., (1997). Fertirrigación. Cultivos hortícolas y ornamentales. Ed. MundiPrensa.

Casas, A. y Casas E., (1999). Análisis de SueloAgua-Planta y su aplicación en la nutrición de cultivos. Ed. Caja Rural de Almería, Almería (España).

Consejería de Agricultura y Pesca, Junta de Andalucía, (2004). Memoria resumen año 2003. Delegación provincial de Almería.

Consejería de Agricultura y Pesca, Junta de Andalucía, (2002). Inventario y caracterización de regadíos de Andalucía. Actualización 2002, CD-ROM.

Contreras, J.I., (2001). Efectos del uso de agua residual depurada sobre suelo y cultivo en invernaderos de Almería. Proyecto Monográfico Ingeniería Técnica Agrícola en Mecanización y Construcciones Rurales, Escuela Politécnica Superior, Universidad de Almería, (España).

Crook, J., (1991). Quality criteria for reclaimed water. Wat. Sci. Tech., 24 (9), 109-121.

Devron, J.I., (1983). Principales fuentes de nitrógeno fijado biológicamente en ecosistemas mayores. Organización de las $\mathrm{Na}$ ciones Unidas para la Agricultura y la Alimentación, Roma (Italia), I Biol. 3, 1-4.

Férnandez, M.D., Orgaz, F., Fereres, E., López, J.C., Céspedes, A., Pérez, J., Bonachela, S. y Gallardo, M., (2000). Programación del riego de cultivos hortícolas bajo invernadero en el sudeste español. Ed. CAJAMAR.

Hita, O., (2002). Efectos de la utilización de acolchados reflectantes sobre el crecimiento, producción y extracción de nutrientes en judía de mata baja en invernadero. Proyecto Monográfico de la Escuela Politécnica Superior, Universidad de Almería (España).
Hussain, G. y Al-Saati, A.J., (1999). Wastewater quality and its reuse in agriculture in Saudi Arabia. Desalination, 123, 241-251.

Kale, C.K. y Bal, A.S., (1987). Reuse of stabilization pond effluent for Citrus reticulata (orange), forest and road verge plants. Water Science and Technology, 19 (12), 307-315.

Kirkham, M.B., (1986). Problems of using wastewater on vegetable crops. HortScience, 21, 24-27.

Koo, R.C.J. y Zekri, M., (1989). Citrus irrigation with reclaimed municipal wastewater. Proc. Flo. Sta. Hort. Soc., 102, 52-56.

Langlais, B., Reckhow, D.A. y Brink, D.R., (1991). Ozone in water treatment application and engineering. Co-operative Research Report, Lewis.

López-Gálvez, J., Téllez, M.M. y Bretones, F., (1991). Efectos de la fertilización nitrogenada sobre la cosecha y la extracción de macronutrientes en un cultivo de judía de enrame. II Congreso Nacional de Ciencias Fertirrigación, 207-212, Almería (España).

M.A.P.A., (1994). Métodos Oficiales de análisis. Tomo III. Secretaría General Técnica del Ministerio de Agricultura, Pesca y Alimentación, Madrid (España).

Maloupa, E., Papadopoulos, A. y Pateras, D., (1999). Wastewater re-use in horticultural crops growing in soil and soilless media. Acta Horticulturae, 481, 603-607.

Martínez Raya, A., (1987). Comportamiento del riego bajo enarenado en invernadero. Balance de salinidad y fertilizantes, en especial de cultivos de pimiento y judía. Tesis Doctoral, Escuela Técnica Superior de Ingenieros Agrónomos, Universidad Politécnica de Madrid.

Martínez, J.L., Cervantes, D., González, E., Valverde, A., Navarrete, F., Molina, L. y Sánchez, F., (1990). Estudio de la contaminación por boro de las aguas de los campos de Níjar y de Dalías y de las cuencas bajas de los ríos Adra y Andarax. Boletín del IEA, 9-10, 235-268.

Maurer, M.A., Davies, F.S. y Graetz, D.A., (1995). Reclaimed wastewater irrigation and fertilization of mature "Redblush" grapefruit trees on spodosols in Florida. The Journal of The American Society For Horticultural Science, 120 (3), 394-402. 
Montserrat, X., (1993). Aplicación de aguas residuales en Sant Jordi (Mallorca). Candela, L. y Varela, M. (eds.), La zona no saturada y la contaminación de las aguas subterráneas. Teoría, Medición y Modelos, Universidad Politécnica de Cataluña, Barcelona (España).

Neilsen, G.H., Stevenson, D.S., Fitzpatrick, J.J. y Brownlee, C.H., (1989). Yield and plant nutrient content of vegetables trickle-irrigated with municipal wastewater. HortScience, 24, 249-252.

Organización Mundial de la Salud, (1989). Health guidelines for the use of wastewater in agriculture and aquaculture. Technology Report, No 778, WHO, Genova.

Page, A.L., Chang, A.C., Sposito, G. y Mattigod, S., (1981). Trace elements in wastewater: Their effects on plant growth and composition and their behavior in soils. Modeling wastewater renovation, Land treatment, Iskandar, I.K. (ed.), John Wiley \& Sons.

Pérez, J.J. y Vallverdú, A., (1997). Depuración y reutilización de aguas residuales. Encuentro Medioambiental Almeriense, Servicio de publicaciones, Universidad de Almería y Caja Rural de Almería (eds.).

Pescod, M.B., (1992). Wastewater treatment and use in agriculture. Irrigation and Drainage, 47, Ed. FAO, Roma (Italia).

Ramos, C., (1997). El uso de aguas residuales en riegos localizados y en cultivos hidropónicos. Fórum Internacional de Horticultura y Tecnología (Fitech), III FITECH - La automatización de la Hidroponía y Fertirrigación, Valencia (España).

Reglamento (CE) 912/2001. Comisión de 10 de mayo de 2001 por el que se establecen las normas de comercialización de las judías.

Rojo, I., Pérez, J.J., Moreno, R., Barón, J., VaIlverdú, A.M. y Segovia, J., (1996). Eficacia microbiológica del tratamiento de aguas residuales con ozono para su uso en riego. Actas del XIV Congreso Nacional de Riegos, 660-667, Almería (España).
Rosas, I., Báez, A. y Coutiño, M., (1984). Bacteriological quality of crops irrigated with wastewater in the Xochimilco plots, Mexico city, Mexico. Applied and Environmental Microbiology, 47 (5), 1074-1079.

Salinas, J.A., (2001). Los recursos hídricos de Almería. En: Problemática de la gestión del agua en regiones semiáridas. Instituto de Estudios Almerienses y Diputación de Almería (eds.), Colección Actas No. 42, 161-166.

Segura, M.L., Granados, M.R. y Contreras, J.I., (2003). Utilización de agua residual depurada para riego de judía verde: sus efectos sobre el cultivo y la fertilidad del suelo. XXI Congreso Nacional de Riegos y Drenajes, Mérida (España).

Segura, M.L., Martínez, S. y Granados, M.R., (2001). Uso del agua residual depurada en el fertirriego del tomate cultivado bajo condiciones de invernadero. IV Congreso Ibérico de Ciencias Hortícolas, Libro de resúmenes 175, Cáceres, 7-11 de mayo.

Segura, M.L., Moreno, R., Martínez, S., Pérez, J. y Moreno, J., (2000). Effects of residual purified irrigation water on melon growth under greenhouse conditions. International Symposium on Protected Cultivation in Mild Winter Climates: Current Trends for Sustainable Technologies. Murcia (España), Acta Horticulturae, 559 (1), 345351.

Shahalam, A., Zahra, B.M.A. y Jaradat, A., (1998). Wastewater irrigation effect on soil, crop and environment: a pilot scale study at Irbid, Jordan. Water, Air and Soil Pollution, 106, 425-428.

Soltanpour, P.N., (1991). Determination of nutrient availability and elemental toxicity by AB-DTPA soil test and ICP. By SpringerVerlag, New York Inc., Advances in Soil Science, 16, 165-190.

Xu, P., Janex, M.L., Savoye, P., Cockx, A., Lazarova, V., (2002). Wastewater disinfection by ozone: main parameters for process desing. Water Research, 36, 1043-1055. 